\title{
Groupoid Actions on Fractafolds ${ }^{\star}$
}

Marius IONESCU ${ }^{\dagger}$ and Alex KUMJIAN ${ }^{\ddagger}$

${ }^{\dagger}$ Department of Mathematics, United States Naval Academy, Annapolis, MD, 21402-5002, USA

E-mail: felijohn@gmail.com

$\ddagger$ Department of Mathematics, University of Nevada, Reno, NV, 89557, USA

E-mail: alex@unr.edu

Received February 04, 2014, in final form June 21, 2014; Published online June 28, 2014

http://dx.doi.org/10.3842/SIGMA.2014.068

\begin{abstract}
We define a bundle over a totally disconnected set such that each fiber is homeomorphic to a fractal blowup. We prove that there is a natural action of a Renault-Deaconu groupoid on our fractafold bundle and that the resulting action groupoid is a RenaultDeaconu groupoid itself. We also show that when the bundle is locally compact the associated $C^{*}$-algebra is primitive and has a densely defined lower-semicontinuous trace.
\end{abstract}

Key words: Renault-Deaconu groupoids; fractafolds; iterated function systems

2010 Mathematics Subject Classification: 28A80; 22A22; 46L55; 46L05

Dedicated to Marc A. Rieffel on the occasion of his 75th birthday

\section{Introduction}

The goal of this paper is to find and analyze symmetries of fractals associated to iterated function systems $\left(F_{1}, \ldots, F_{N}\right)$ and to study the associated $C^{*}$-algebras that might arise from the dynamics. In [19] Stricharz constructed a family of fractafold blowups of the invariant set of an iterated function system which is parameterized by infinite words in the alphabet $\{1, \ldots, N\}$ and observed that two such blowups are naturally homeomorphic if the parametrizing words are eventually the same. We endow these fractafold blowups with the inductive limit topology and assemble them into a fractafold bundle $L$.

In general there do not appear to be any natural nontrivial symmetries of a generic blowup but Stricharz's observation suggests that we look for symmetries of the bundle instead. Indeed we show that the homeomorphisms between fibers observed by Stricharz give rise to a natural groupoid action on $L$, the fractafold bundle. This groupoid action and the associated action groupoid $\tilde{\mathcal{G}}$ constitute the main focus for this work.

We prove that the there is a local homeomorphism $\tilde{\sigma}$ on $L$ such that $\tilde{\mathcal{G}}$ is isomorphic to the Renault-Deaconu groupoid associated to $\tilde{\sigma}$ and, in particular, $\tilde{\mathcal{G}}$ is étale. We also prove that $\tilde{\mathcal{G}}$ is topologically principal and has a dense orbit. If the iterated function system satisfies the open set condition then we construct a $\tilde{\mathcal{G}}$-invariant measure on the unit space $\tilde{\mathcal{G}}^{0}$.

Now suppose that $L$ is locally compact. Then the associated $C^{*}$-algebra, $C^{*}(\tilde{\mathcal{G}})$, is primitive. If, in addition the iterated function system satisfies the open set condition, then the associated $C^{*}$-algebra has a densely defined lower semi-continuous trace.

We begin by reviewing some of the background material and by proving some general results about Renault-Deaconu groupoids in Section 2. These results will be useful in our analysis of the fractafold bundle $L$ and the associated action groupoid $\tilde{\mathcal{G}}$ in Section 3 . In the final section we consider some examples to illustrate the theory. We show that the action groupoid is not

\footnotetext{
${ }^{\star}$ This paper is a contribution to the Special Issue on Noncommutative Geometry and Quantum Groups in honor of Marc A. Rieffel. The full collection is available at http://www.emis.de/journals/SIGMA/Rieffel.html
} 
in general minimal. We also point out cases when the fractafold bundle $L$ fails to be locally compact.

\section{Renault-Deaconu groupoid and groupoid actions}

In this section we prove that if $\mathcal{G}$ is the Renault-Deaconu groupoid associated to a local homeomorphism $\sigma$ on a topological space $X$ such that $\mathcal{G}$ acts on a topological space $Z$, then $\sigma$ lifts to a natural local homeomorphism $\tilde{\sigma}$ on $Z$. Moreover, we show that the resulting action groupoid is isomorphic to the Renault-Deaconu groupoid associated to $\tilde{\sigma}$. We begin by reviewing some of the background material that we need. While some of the motivation for this work came from the theory of $C^{*}$-algebras associated to groupoids, many of our results hold for topological groupoids that are not necessarily locally compact. Since some of the groupoids in our examples are not locally compact, the only generic assumption on the topological spaces and topological groupoids that we make is that they are Hausdorff.

Let $G$ be a Hausdorff topological groupoid (see [18], cf. also [16]). Then the structure maps are continuous and, in addition, both the range map $r$ (where $r(x)=x x^{-1}$ ) and the source map $s$ (where $s(x)=x^{-1} x$ ) are open. We write $G^{0}$ for the unit space of $G$. The groupoid $G$ is said to be étale if $s$ is a local homeomorphism (or equivalently, $r$ is a local homeomorphism). A subset $S \subset G$ is called a $G$-set or a bisection if the restrictions, $\left.r\right|_{S},\left.s\right|_{S}$, are both injective. If $G$ is étale, then it has a cover of open $G$-sets and the restriction of either $r$ or $s$ to an open $G$-set is a homeomorphism onto an open subset of $G^{0}$.

Let $G$ be topological groupoid. If the set of points in $G^{0}$ with trivial isotropy,

$$
\left\{x \in G^{0} \mid s(\gamma)=r(\gamma)=x \text { only if } \gamma=x\right\}
$$

is dense in $G^{0}$, we say that $G$ is topologically principal (see [18, Definition 3.5(ii)]).

If $G$ is a locally compact étale groupoid we let $C^{*}(G)$ denote the full $C^{*}$-algebra of $G$ and $C_{r}^{*}(G)$ denote the reduced $C^{*}$-algebra of $G$. If, in addition, $G$ is amenable ${ }^{1}$ the canonical quotient map $C^{*}(G) \rightarrow C_{r}^{*}(G)$ is an isomorphism. We regard $C_{0}\left(G^{0}\right)$ as an abelian $C^{*}$-subalgebra of both.

Let $X$ be a topological space and let $\sigma: X \rightarrow X$ be a local homeomorphism on $X$. The Renault-Deaconu groupoid associated to $\sigma[1,7,8,16,17]$ is

$$
\mathcal{G}=\mathcal{G}(X, \sigma)=\left\{(x, m-n, y) \in X \times \mathbb{Z} \times X: \sigma^{m}(x)=\sigma^{n}(y)\right\} .
$$

Two elements $(x, m, y)$ and $(z, n, w)$ in $\mathcal{G}$ are composable if and only if $y=z$ and, in this case, their product is

$$
(x, m, y)(y, n, w)=(x, m+n, w) .
$$

The inverse of an element in $\mathcal{G}$ is defined by

$$
(x, n, y)^{-1}=(y,-n, x) .
$$

Thus the range and source maps are given by $r(x, n, y)=(x, 0, x)$ and $s(x, n, y)=(y, 0, y)$. Hence $\mathcal{G}^{0}$, the unit space of $\mathcal{G}$, may be identified with $X$ via the map $(x, 0, x) \mapsto x$ (in the sequel we will often make this identification without comment). A basis for the topology consists of sets of the form

$$
\mathcal{G}(U, m, n, V)=\left\{(x, m-n, y): \sigma^{m}(x)=\sigma^{n}(y), x \in U, y \in V\right\},
$$

\footnotetext{
${ }^{1}$ See [2, Proposition 2.2.13] for different characterizations of amenability for topological groupoids.
} 
where $m$ and $n \in \mathbb{N}, U$ and $V$ are open subsets of $X$ such that both $\left.\sigma^{m}\right|_{U}$ and $\left.\sigma^{n}\right|_{V}$ are injective and $\sigma^{m}(U)=\sigma^{n}(V)$. Note that the range map $r$ induces a homeomorphism $\mathcal{G}(U, m, n, V) \cong U$. Hence, with this topology $\mathcal{G}$ is an étale groupoid. If $X$ is locally compact then $\mathcal{G}=\mathcal{G}(X, \sigma)$ is a locally compact groupoid.

Let $G$ be a topological groupoid with unit space $G^{0}=X$. Let $Z$ be a topological space and let $\rho: Z \rightarrow X$ be a continuous open map. A continuous map $\alpha: G * Z \rightarrow Z$ (where $G * Z=\{(\gamma, z) \mid s(\gamma)=\rho(z)\})$, write $\alpha(\gamma, z)=\gamma \cdot z$, such that $\rho(\gamma \cdot z)=r(\gamma), \gamma_{2} \cdot\left(\gamma_{1} \cdot z\right)=\left(\gamma_{2} \gamma_{1}\right) \cdot z$, and $\rho(z) \cdot z=z$, for all $\gamma, \gamma_{1}, \gamma_{2} \in G$ and $z \in Z$ is said to be a left action of $G$ on $Z$ (see [13]). Then $G * Z$ may itself be endowed with the structure of a topological groupoid where the topology is inherited from $G \times Z$; it is called the (left) action groupoid (associated to $\alpha$ ). If $G$ and $Z$ are locally compact, then so is $G * Z$. Recall that $\left(\gamma_{1}, z_{1}\right)$ and $\left(\gamma_{2}, z_{2}\right) \in G * Z$ are composable if $z_{1}=\gamma_{2} \cdot z_{2}$ and the product is given by $\left(\gamma_{1}, z_{1}\right)\left(\gamma_{2}, z_{2}\right)=\left(\gamma_{1} \gamma_{2}, z_{2}\right)$. The inverse of an element $(\gamma, z)$ is $\left(\gamma^{-1}, \gamma \cdot z\right)$. Therefore $(G * Z)^{0}=\{(\rho(z), z) \mid z \in Z\}$ and thus the unit space may be identified with $Z$ using the projection onto the second factor $(\rho(z), z) \mapsto z$; note that $s(\gamma, z)=(s(\gamma), z)$ and $r(\gamma, z)=(r(\gamma), \gamma \cdot z)$. If $V$ is an open subset of $G$ and $W$ is an open subset of $Z$, then $V * W:=(V \times W) \cap(G * Z)$ is an open subset of $G * Z$.

Lemma 2.1. With notation as above let $\alpha: G * Z \rightarrow Z$ be a left action and suppose that $G$ is étale. Then the action groupoid $G * Z$ is itself étale and the action map $\alpha: G * Z \rightarrow Z$ is open.

Proof. Let $\left(\gamma_{0}, z_{0}\right) \in G * Z$ and let $V$ be an open $G$-set containing $\gamma_{0}$. Then $\left.s\right|_{V}$ yields a homeomorphism $V \cong s(V)$. Set $W:=\rho^{-1}(s(V))$ and observe that $V * W$ is an open neighborhood of $\left(\gamma_{0}, z_{0}\right)$. Then since $s(\gamma, z)=(s(\gamma), z)$, we have $s(V * W)=s(V) * W=\rho(W) * W$ is open and the restriction $\left.s\right|_{V * W}$ is a continuous injection; the inverse map $(\rho(z), z) \mapsto\left(\left(\left.s\right|_{V}\right)^{-1}(\rho(z)), z\right)$ is continuous. Hence, $\left.s\right|_{V * W}$ is a homeomorphism onto an open subset of $(G * Z)^{0}$. So $s: G * Z \rightarrow$ $(G * Z)^{0}$ is a local homeomorphism and $G * Z$ is étale.

Let $\pi: G * Z \rightarrow Z$ be the projection map onto the second factor and observe that $\alpha=\pi \circ r$. Then since $r(G * Z)=(G * Z)^{0}$ is open and both $\pi$ and $r$ are open maps, the action map $\alpha: G * Z \rightarrow Z$ is open.

We next show that if the groupoid acting is a Renault-Deaconu groupoid the action groupoid is itself of the same type.

Theorem 2.2. Let $\sigma$ be a local homeomorphism on a topological space $X$ and suppose that the Renault-Deaconu groupoid $\mathcal{G}=\mathcal{G}(X, \sigma)$ acts on the left on the topological space $Z$. Define $\tilde{\sigma}: Z \rightarrow Z$ via

$$
\tilde{\sigma}(z)=(\sigma(\rho(z)),-1, \rho(z)) \cdot z .
$$

Then $\tilde{\sigma}$ is a local homeomorphism on $Z$ such that $\rho \circ \tilde{\sigma}=\sigma \circ \rho$. Moreover, the left action groupoid $\mathcal{G} * Z$ is isomorphic to $\tilde{\mathcal{G}}=\mathcal{G}(Z, \tilde{\sigma})$, the Renault-Deaconu groupoid associated to $\tilde{\sigma}$, via the map $\Psi: \tilde{\mathcal{G}} \rightarrow \mathcal{G} * Z$ given by

$$
\Psi((t, m-n, s))=((\rho(t), m-n, \rho(s)), s),
$$

where $(t, m-n, s) \in \tilde{\mathcal{G}} \subset Z \times \mathbb{Z} \times Z$.

Proof. Let $z \in Z$. We need to find an open neighborhood $U$ of $z$ such that $\tilde{\sigma}(U)$ is open and $\left.\tilde{\sigma}\right|_{U}: U \rightarrow \tilde{\sigma}(U)$ is a homeomorphism. Since $\sigma$ is a local homeomorphism there is an open neighborhood $V \subset X$ of $\rho(z)$ such that $\sigma(V)$ is open and $\left.\sigma\right|_{V}: V \rightarrow \sigma(V)$ is a homeomorphism. Let $U=\rho^{-1}(V)$. We prove first that $\left.\tilde{\sigma}\right|_{U}$ is one-to-one. Let $z_{1}, z_{2} \in U$ such that $\tilde{\sigma}\left(z_{1}\right)=\tilde{\sigma}\left(z_{2}\right)$. This means, by definition, that

$$
\left(\sigma\left(\rho\left(z_{1}\right)\right),-1, \rho\left(z_{1}\right)\right) \cdot z_{1}=\left(\sigma\left(\rho\left(z_{2}\right)\right),-1, \rho\left(z_{2}\right)\right) \cdot z_{2} .
$$


Since $\rho\left(\left(\sigma\left(\rho\left(z_{1}\right)\right),-1, \rho\left(z_{1}\right)\right) \cdot z_{1}\right)=\sigma\left(\rho\left(z_{1}\right)\right)$ and $\rho\left(\left(\sigma\left(\rho\left(z_{2}\right)\right),-1, \rho\left(z_{2}\right)\right) \cdot z_{2}\right)=\sigma\left(\rho\left(z_{2}\right)\right)$, and since $\rho\left(z_{1}\right)$ and $\rho\left(z_{2}\right)$ are elements of $V$, it follows that $\rho\left(z_{1}\right)=\rho\left(z_{2}\right)$. Moreover, if we multiply the equation (2.2) on the left by

$$
\left(\sigma\left(\rho\left(z_{1}\right)\right),-1, \rho\left(z_{1}\right)\right)^{-1}=\left(\rho\left(z_{1}\right), 1, \sigma\left(\rho\left(z_{1}\right)\right)\right),
$$

we obtain that

$$
\begin{aligned}
z_{1} & =\left(\rho\left(z_{1}\right), 1, \sigma\left(\rho\left(z_{1}\right)\right)\right) \cdot\left(\left(\sigma\left(\rho\left(z_{2}\right)\right),-1, \rho\left(z_{2}\right)\right) \cdot z_{2}\right) \\
& =\left(\rho\left(z_{1}\right), 0, \rho\left(z_{2}\right)\right) \cdot z_{2}=\left(\rho\left(z_{2}\right), 0, \rho\left(z_{2}\right)\right) \cdot z_{2}=z_{2} .
\end{aligned}
$$

Thus $\left.\tilde{\sigma}\right|_{U}$ is one-to-one. Observe that $\rho(U)=V$ and $\sigma(V)$ are both open. Hence, $\mathcal{G}(\sigma(V), 0,1$, $V) * U$ is open. Since the action map $\alpha$ is open by Lemma 2.1,

$$
\tilde{\sigma}(U)=\alpha(\mathcal{G}(\sigma(V), 0,1, V) * U)
$$

is open. Therefore $\tilde{\sigma}$ is a local homeomorphism. The equation $\rho \circ \tilde{\sigma}=\sigma \circ \rho$ is an easy computation.

We now prove the second part of the theorem, namely that $\Psi: \tilde{\mathcal{G}} \rightarrow \mathcal{G} * Z$ (see formula (2.1)) is an isomorphism where $\tilde{\mathcal{G}}=\mathcal{G}(Z, \tilde{\sigma})$ is the Renault-Deaconu groupoid associated to $\tilde{\sigma}$, that is,

$$
\tilde{\mathcal{G}}=\left\{(t, m-n, s) \in Z \times \mathbb{Z} \times Z: \tilde{\sigma}^{m}(t)=\tilde{\sigma}^{n}(s)\right\} .
$$

We first show that $\Psi$ is well defined. Let $(t, m-n, s) \in \tilde{\mathcal{G}}$; then $\tilde{\sigma}^{m}(t)=\tilde{\sigma}^{n}(s)$. An easy computation shows that $\tilde{\sigma}^{m}(t)=\left(\sigma^{m}(\rho(t)),-m, \rho(t)\right) \cdot t$. Thus, if $\tilde{\sigma}^{m}(t)=\tilde{\sigma}^{n}(s)$ then $\sigma^{m}(\rho(t))=$ $\sigma^{n}(\rho(s))$ and, moreover, $t=(\rho(t), m-n, \rho(s)) \cdot s$. Hence $(\rho(t), m-n, \rho(s)) \in \mathcal{G}$ and $\Psi$ is well defined.

We next show that $\Psi$ is a groupoid morphism. Let $\left(t_{1}, n_{1}, s_{1}\right)$ and $\left(t_{2}, n_{2}, s_{2}\right)$ be two composable elements in $\tilde{\mathcal{G}}$. Then $s_{1}=t_{2}$ and

$$
\left(t_{1}, n_{1}, s_{1}\right)\left(t_{2}, n_{2}, s_{2}\right)=\left(t_{1}, n_{1}+n_{2}, s_{2}\right) .
$$

Therefore

$$
\Psi\left(\left(t_{1}, n_{1}, s_{1}\right)\left(t_{2}, n_{2}, s_{2}\right)\right)=\left(\left(\rho\left(t_{1}\right), n_{1}+n_{2}, \rho\left(s_{2}\right)\right), s_{2}\right) .
$$

Moreover, since $s_{1}=t_{2}=\left(\rho\left(t_{2}\right), n_{2}, \rho\left(s_{2}\right)\right) \cdot s_{2}$, it follows that $\left(\left(\rho\left(t_{1}\right), n_{1}, \rho\left(s_{1}\right)\right), s_{1}\right)$ and $\left(\left(\rho\left(t_{2}\right), n_{2}\right.\right.$, $\left.\left.\rho\left(s_{2}\right)\right), s_{2}\right)$ are composable and we have that

$$
\begin{aligned}
\Psi\left(\left(t_{1}, n_{1}, s_{1}\right)\right) \Psi\left(\left(t_{2}, n_{2}, s_{2}\right)\right) & =\left(\left(\rho\left(t_{1}\right), n_{1}, \rho\left(s_{1}\right)\right), s_{1}\right) \cdot\left(\left(\rho\left(t_{2}\right), n_{2}, \rho\left(s_{2}\right)\right), s_{2}\right) \\
& =\left(\left(\rho\left(t_{1}\right), n_{1}+n_{2}, \rho\left(s_{2}\right)\right), s_{2}\right) .
\end{aligned}
$$

Equations (2.3) and (2.4) imply that $\Psi$ is a groupoid morphism.

Next we prove that $\Psi$ is one-to-one and onto. Let $\left(t_{1}, n_{1}, s_{1}\right)$ and $\left(t_{2}, n_{2}, s_{2}\right) \in \tilde{\mathcal{G}}$ such that $\Psi\left(\left(t_{1}, n_{1}, s_{1}\right)\right)=\Psi\left(\left(t_{2}, n_{2}, s_{2}\right)\right)$. Thus

$$
\left(\left(\rho\left(t_{1}\right), n_{1}, \rho\left(s_{1}\right)\right), s_{1}\right)=\left(\left(\rho\left(t_{2}\right), n_{2}, \rho\left(s_{2}\right)\right), s_{2}\right) .
$$

Therefore $s_{1}=s_{2}, \rho\left(t_{1}\right)=\rho\left(t_{2}\right)$, and $n_{1}=n_{2}$. Moreover

$$
t_{1}=\left(\rho\left(t_{1}\right), n_{1}, \rho\left(s_{1}\right)\right) \cdot s_{1}=\left(\rho\left(t_{2}\right), n_{2}, \rho\left(s_{2}\right)\right) \cdot s_{2}=t_{2} .
$$

Hence $\Psi$ is one-to-one. To see that $\Psi$ is onto, let $((x, n, y), s) \in \mathcal{G} * Z$, and recall that $y=\rho(s)$. Let $t=(x, n, y) \cdot s$. Then $\rho(t)=x$ and

$$
\Psi(t, n, s)=((\rho(t), n, \rho(s)), s)=((x, n, y), s) .
$$


To show that $\Psi$ is continuous, let $U=\mathcal{G}\left(U_{1}, m, n, U_{2}\right) * V_{2}$ be an open set in $\mathcal{G} * Z$ such that $U_{1}, U_{2}$ are open subsets of $X, \sigma^{m}\left(U_{1}\right)=\sigma^{n}\left(U_{2}\right)$ is open, $\left.\sigma^{m}\right|_{U_{1}}: U_{1} \rightarrow \sigma^{m}\left(U_{1}\right)$ and $\left.\sigma^{n}\right|_{U_{2}}: U_{2} \rightarrow \sigma^{n}\left(U_{2}\right)$ are homeomorphisms, and $V_{2}=\rho^{-1}\left(U_{2}\right)$. Then

$$
\Psi^{-1}(U)=\left(\rho^{-1}\left(U_{1}\right), m, n, \rho^{-1}\left(U_{2}\right)\right) .
$$

One can repeat the argument that we made when showing that $\tilde{\sigma}$ is a local homeomorphism and prove that $\tilde{\sigma}^{m}\left(\rho^{-1}\left(U_{1}\right)\right)$ is open and $\left.\tilde{\sigma}^{m}\right|_{\rho^{-1}\left(U_{1}\right)}: \rho^{-1}\left(U_{1}\right) \rightarrow \tilde{\sigma}^{m}\left(\rho^{-1}\left(U_{1}\right)\right)$ is a homeomorphism, and, similarly, $\tilde{\sigma}^{n}\left(\rho^{-1}\left(U_{2}\right)\right)$ is open and $\left.\tilde{\sigma}^{n}\right|_{\rho^{-1}\left(U_{2}\right)}: \rho^{-1}\left(U_{2}\right) \rightarrow \tilde{\sigma}^{n}\left(\rho^{-1}\left(U_{2}\right)\right)$ is a homeomorphism. Notice that $\tilde{\sigma}^{m}(t)=\tilde{\sigma}^{n}(s)$ with $t \in \rho^{-1}\left(U_{1}\right)$ and $s \in \rho^{-1}\left(U_{2}\right)$ if and only if $\sigma^{m}(\rho(t))=\sigma^{n}(\rho(s))$. Since $\sigma^{m}\left(U_{1}\right)=\sigma^{n}\left(U_{2}\right)$ it follows that $\tilde{\sigma}^{m}\left(\rho^{-1}\left(U_{1}\right)\right)=\tilde{\sigma}^{n}\left(\rho^{-1}\left(U_{2}\right)\right)$. Therefore $\Psi^{-1}(U)$ is an open cylinder in $\tilde{\mathcal{G}}$ and $\Psi$ is continuous. Finally, $\Psi^{-1}$ is continuous since it is a composition of continuous maps.

For the rest of this section we assume that both $X$ and $Z$ are locally compact spaces. This assumption implies that both $\mathcal{G}$ and $\tilde{\mathcal{G}}$ are locally compact groupoids.

Corollary 2.3. Assume that $\sigma$ is a local homeomorphism on a locally compact space $X$ and assume that the Renault-Deaconu groupoid $\mathcal{G}=\mathcal{G}(X, \sigma)$ acts on the locally compact space $Z$. The groupoid $\tilde{\mathcal{G}} \cong \mathcal{G} * Z$ is amenable and the full and reduced $C^{*}$-algebra of $\mathcal{G} * Z$ coincide. Moreover, $C^{*}(\mathcal{G} * Z)$ is nuclear.

Proof. This is an immediate consequence of [17, Proposition 2.9].

As another application of Theorem 2.2, we can identify $C^{*}(\mathcal{G} * Z)$ as a Cuntz-Pimsner algebra [15]. The closure of $C_{c}(Z)$ under a suitable norm may be viewed as a $C^{*}$-correspondence $\mathcal{Z}$ over the $C^{*}$-algebra $C_{0}(Z)$. The inner product is defined for $\xi, \eta \in C_{c}(Z)$ by

$$
\langle\xi, \eta\rangle(z)=\sum_{\tilde{\sigma}(y)=z} \overline{\xi(y)} \eta(y)
$$

the left and right actions of $C_{0}(Z)$ are given by $(a \cdot \xi \cdot b)(z)=a(z) \xi(z) b(\tilde{\sigma}(z))$ where $a, b \in C_{0}(Z)$ and $\xi \in C_{c}(Z)$. The following corollary follows from [8, Theorem 7 .

Corollary 2.4. Under the hypothesis of Corollary 2.3, the groupoid $C^{*}$-algebra $C^{*}(\mathcal{G} * Z)$ is isomorphic to the Cuntz-Pimsner $C^{*}$-algebra $\mathcal{O}_{\mathcal{Z}}$.

\section{Groupoid actions on fractafolds}

In this section we build a fractafold bundle $L$ such that the Renault-Deaconu $\mathcal{G}$ groupoid associated to a one-sided shift map $\sigma$ acts naturally on $L$. The action groupoid $\mathcal{G} * L$ encapsulates the symmetries of the fractafold bundle. Using the results of the previous section, $\sigma$ extends to a local homeomorphism $\tilde{\sigma}$ on $L$. We prove that $\tilde{\sigma}$ is essentially free and, hence, $\mathcal{G} * L$ is topologically principal. Moreover, we show that the action groupoid $\mathcal{G} * L$ contains a dense orbit. If $L$ is locally compact, it follows that $C^{*}(\mathcal{G} * L)$ is primitive. If the iterated function system defining $L$ satisfies the open set condition, then we build a $\mathcal{G} * L$-invariant measure on $L$. Thus if $L$ is, in addition, locally compact, then $C^{*}(\mathcal{G} * L)$ has a densely defined lower semicontinuous trace.

Let $(T, d)$ be a complete metric space and let $\left(F_{1}, \ldots, F_{N}\right)$ be an iterated function system on $T[3,9,11]$. That is, each $F_{i}$ is a strict contraction on $T$. We assume that the iterated function system is non-degenerate, meaning that there are constants $0<r_{i} \leq R_{i}<1, i=1, \ldots, N$, such that

$$
r_{i} d\left(t, t^{\prime}\right) \leq d\left(F_{i}(t), F_{i}\left(t^{\prime}\right)\right) \leq R_{i} d\left(t, t^{\prime}\right) \quad \text { for all } \quad t, t^{\prime} \in T, \quad i=1, \ldots, N .
$$


We further assume map $F_{i}$ is surjective for all $i=1, \ldots, N$; so the $F_{i}$ are homeomorphisms. If we can chose $r_{i}=R_{i}$ in (3.1), then $F_{i}$ is a similarity (or a similitude). For an iterated function system there is a unique compact invariant set $K$ [11, Theorem 3.1.3] such that

$$
K=F_{1}(K) \cup \cdots \cup F_{N}(K) .
$$

If $F_{i}(K) \bigcap F_{j}(K)=\varnothing$ for all $i \neq j$, then the iterated function system is called totally disconnected. In this case the invariant set $K$ is a totally disconnected set [3, Theorem 8.2.1]. If $N \geq 3$, using [11, Remark 3.1.9] one can find examples of iterated function systems that are not totally disconnected but have a totally disconnected invariant set.

Let $W=\{1, \ldots, N\}$ and define $W^{*}=\bigcup_{n \in \mathbb{N}} W^{n}$ to be the set of finite words over the alphabet $W$, and $X=W^{\infty}$ to be the set of infinite words (sequences) with elements in $W$. If $\omega \in W^{n}$ we say that the length of $\omega$, denoted by $|\omega|$, is $n$. For $\omega \in W^{n}$ we write $F_{\omega}(x):=$ $F_{\omega_{n}} \circ \cdots \circ F_{\omega_{1}}(x), F_{\omega}^{-1}(x):=F_{\omega_{1}}^{-1} \circ \cdots \circ F_{\omega_{n}}^{-1}(x), r_{\omega}:=r_{\omega_{1}} \cdots r_{\omega_{n}}$ and similarly for $R_{\omega}$.

If $\omega \in W^{n}$ or $x \in X$ and if $k \in \mathbb{N}$ such that $k \leq n$ (if applicable), then we write

$$
\omega(k):=\omega_{1} \cdots \omega_{k} \quad \text { and } \quad x(k):=x_{1} \cdots x_{k} .
$$

Given a finite sequence $\omega \in W^{n}$ or an infinite sequence $x \in X$ set

$$
L_{n}(\omega)=F_{\omega(n)}^{-1}(K) \quad \text { and } \quad L_{n}(x):=L_{n}(x(n))
$$

Then, if $x \in X, L_{n}(x) \subset L_{n+1}(x)$ and we define $L(x):=\bigcup_{n \in \mathbb{N}} L_{n}(x)$ endowed with the inductive limit topology. We refer to $L(x)$ as the infinite blow-up of $K$ at $x$. Our definition is not quite the same as the one used by Stricharz since he uses the relative topology (see [19] and [20, Chapter 5.4]); but the two seem to agree in some cases, for example, when the blowups are zero dimensional. Two blow-ups $L(x)$ and $L(y)$ are homeomorphic if the infinite sequences $x$ and $y$ differ in a finite number of indices. For example, if $y=x_{2} x_{3} \cdots, F_{x_{1}}$ extends to a homeomorphism from $L(x)$ to $L(y)$.

If $U$ is a subset of $K$ and $\omega \in W^{n}$ is a finite sequence or $x \in X$ is an infinite word, then we write $L_{n}^{\omega}(U)$ for $F_{\omega(n)}^{-1}(U)$ and $L_{n}^{x}(U)$ for $L_{n}^{x(n)}(U)$.

If $\omega \in W^{*}$, then the clopen cylinder $Z(\omega) \subset X=W^{\infty}$ is defined via

$$
Z(\omega)=\left\{x \in X: x_{i}=\omega_{i}, i=1, \ldots,|\omega|\right\} .
$$

The collection $\{Z(\omega)\}_{\omega \in W^{*}}$ forms a basis of a topology on $X$, and, endowed with this topology, $X$ is a totally disconnected compact space. Moreover, the shift map $\sigma: X \rightarrow X$ defined by $\sigma\left(x_{1} x_{2} \cdots\right)=\left(x_{2} x_{3} \cdots\right)$ is a local homeomorphism on $X$. We write $\mathcal{G}$ for the Renault-Deaconu groupoid associated to $\sigma$ as in Section 2. Recall that the unit space $\mathcal{G}^{0}$ is homeomorphic to $X$.

Next we build a fractafold bundle on which the groupoid $\mathcal{G}$ acts. For $n \geq 0$ define

$$
L_{n}=\bigsqcup_{\omega \in W^{n}} Z(\omega) \times L_{n}(\omega) \subset X \times Y .
$$

Then each $L_{n}$ is a compact space and $L_{n} \subset L_{n+1}$. Observe that that $L_{0}=X \times K$. We define the fractafold bundle $L$ to be the increasing union of $L_{n}, L=\bigcup_{n \geq 0} L_{n}$, endowed with the inductive limit topology. That is, a set $U \subset L$ is open if and only if $U \cap L_{n}$ is open for all $n \geq 0$. We will show below that the base space is $X$. The following characterization of open sets is used later in this section.

Lemma 3.1. Let $x \in X$ and $n \geq 0$. Then $U \subset L_{n}(x)$ is open if and only if there is an open set $V \subset K$ such that $U=L_{n}^{x}(V)$. 
Proof. Recall that the maps $F_{i}, i=1, \ldots, N$, are homeomorphisms. Therefore the maps $F_{\omega}$ and $F_{\omega}^{-1}$ are homeomorphisms for all $\omega \in W^{*}$. The conclusion of the lemma follows immediately.

Recall that, in general, the inductive limit of an increasing sequence of Hausdorff spaces might fail to be Hausdorff. However, as we prove in the following lemma, the bundle $L$ is Hausdorff.

Lemma 3.2. The bundle $L$ endowed with the inductive limit topology as above is a Hausdorff space.

Proof. Let $\iota: L \rightarrow X \times T$ be the inclusion map. If $U$ is an open set in $X \times T$, then $\iota^{-1}(U) \cap L_{n}=$ $U \cap L_{n}$ is open in $L_{n}$ for all $n \geq 1$. Therefore $\iota$ is a continuous one-to-one map. Since $X \times T$ is Hausdorff, it follows that $L$ is Hausdorff as well.

Before we define the action of the Renault-Deaconu groupoid on $L$ we show that the natural projection from $L$ into $X$ is an open map.

Lemma 3.3. The map $\pi: L \rightarrow X, \pi(x, t)=x$ is a continuous open map. Moreover, $\pi^{-1}(\{x\})$ is homeomorphic to the fractafold $L(x)$.

Proof. Since the restriction of $\pi$ to $L_{n}$ is continuous for each $n$ and $L$ is endowed with the inductive limit topology, $\pi$ is also continuous. Let $U$ be an open set in $L$. Therefore $U_{n}:=U \cap L_{n}$ is open for all $n \geq 0$. Hence $U_{n} \cap\left(Z(\omega) \times L_{n}(\omega)\right)$ is open for all $\omega \in W^{n}$. For $\omega \in W^{n}$, the map $\left.\pi\right|_{Z(\omega) \times L_{n}(\omega)}: Z(\omega) \times L_{n}(\omega) \rightarrow Z(\omega)$ is open because it is just the projection onto the first coordinate. Since $Z(\omega)$ is an open subset of $X$ it follows that $\pi\left(U_{n} \cap\left(Z(\omega) \times L_{n}(\omega)\right)\right)$ is open in $X$ for all $\omega \in W^{n}$ and $n \geq 0$. Therefore, $\pi\left(U_{n}\right)$ is open in $X$ for all $n \geq 0$, and hence $\pi(U)=\bigcup_{n} \pi\left(U_{n}\right)$ is open. Thus $\pi$ is an open map. The final assertion is obvious.

The next results shows that the Renault-Deaconu groupoid $\mathcal{G}$ associated to the shift map on $X$ acts on the left on the space $L$. Note that if $\gamma=(x, m-n, y) \in \mathcal{G}$ and $(z, t) \in L$, then $s(\gamma)=\pi(z, t)$ if and only if $y=z$. Let

$$
\mathcal{G} * L=\{((x, m-n, y),(y, t)):(x, m-n, y) \in \mathcal{G},(y, t) \in L\} .
$$

Theorem 3.4. With the notation as above, the Renault-Deaconu groupoid $\mathcal{G}$ associated to the shift map $\sigma$ on $X$ acts on the fractafold bundle $L$ via the map $((x, m-n, y),(y, t)) \mapsto(x, m-$ $n, y) \cdot(y, t)$ defined on $\mathcal{G} * L$, where

$$
(x, m-n, y) \cdot(y, t)=\left(x, F_{x(m)}^{-1} \circ F_{y(n)}(t)\right) .
$$

Moreover, the action map is open.

Proof. We need to prove that the above map is well defined; that is, we need to show that the range of the map is $L$. Let $(x, m-n, y) \in \mathcal{G}$ and $(y, t) \in L$. Let $k \geq 0$ such that $(y, t) \in L_{k}$. Then there is $\omega \in W^{k}$ such that $y \in Z(\omega)$ and $t \in L_{k}(\omega)$. Therefore $y_{i}=\omega_{i}$ for $i=1, \ldots, k$. Notice that it suffices to assume that $n \geq k$. Indeed, if $m^{\prime}-n^{\prime}=m-n$ and $\sigma^{m^{\prime}}(x)=\sigma^{n^{\prime}}(y)$ with $m^{\prime}>m$ and $n^{\prime}>n$, then $x_{m+i}=y_{n+i}$ for all $i=1, \ldots, m^{\prime}-m$ and so $F_{x(m)}^{-1} \circ F_{y(n)}(t)=F_{x\left(m^{\prime}\right)}^{-1} \circ F_{y\left(n^{\prime}\right)}(t)$. Then

$$
F_{y(n)}(t) \in K
$$

and, thus, $F_{x(m)}^{-1} \circ F_{y(n)}(t) \in L_{m}(\alpha)$, where $\alpha=x(m)$. Hence

$$
\left(x, F_{x(m)}^{-1} \circ F_{y(n)}(t)\right) \in Z(\alpha) \times L_{m}(\alpha) \subset L .
$$

So the action is well defined. 
The left action is continuous since for fixed $m$ and $n$ in $\mathbb{N}$ and fixed words $\alpha \in W^{m}$ and $\beta \in W^{n}$, we have that the map $F_{\alpha}^{-1} \circ F_{\beta}$ is continuous. Thus the Renault-Deaconu groupoid $\mathcal{G}$ acts on the left on $L$. The last part of the theorem is an immediate consequence of Lemma 2.1 .

Corollary 3.5. The map $\tilde{\sigma}: L \rightarrow L$ defined by $\tilde{\sigma}(x, t)=\left(\sigma(x), F_{x_{1}}(t)\right)$ is a local homeomorphism on $L$ such that $\pi \circ \tilde{\sigma}=\sigma \circ \pi$.

Proof. Theorems 2.2 and 3.4 imply that the shift map $\sigma$ on $X$, which is a local homeomorphism, lifts to a local homeomorphism $\tilde{\sigma}$ on $L$ such that $\tilde{\sigma}(x, t)=(\sigma(x),-1, x) \cdot(x, t)$. By equation (3.3), $\tilde{\sigma}(x, t)=\left(\sigma(x), F_{x_{1}}(t)\right)$.

Corollary 3.6. Let $\tilde{\sigma}$ be the local homeomorphism on $L$ provided by Corollary 3.5. Then the left action groupoid $\mathcal{G} * L$ is homeomorphic to the Renault-Deaconu groupoid $\tilde{\mathcal{G}}$ associated to $\tilde{\sigma}$.

Proof. This is an immediate consequence of the second part of Theorem 2.2.

Recall from [7, Definition on p. 1781] that a local homeomorphism $\tau$ on a topological space $Z$ is essentially free if

$$
\left\{z \in Z \mid \forall k, l \geq 0, \tau^{k}(z)=\tau^{l}(z) \Rightarrow k=l\right\}
$$

is dense in $Z$. If $\tau$ is essentially free then the Renault-Deaconu groupoid $\mathcal{G}(Z, \tau)$ is topologically principal because, for $z \in Z$, the isotropy group $\mathcal{G}(z)$ is nontrivial if and only if there are $k, l \geq 0$ with $k \neq l$ such that $\tau^{k}(z)=\tau^{l}(z)$ [1, Example 1.2c]. It is easy to see that the shift map $\sigma$ on $X$ is essentially free [7, Example 2].

Proposition 3.7. The local homeomorphism $\tilde{\sigma}$ on L defined in Corollary 3.5 is essentially free. Hence, the Renault-Deaconu groupoid $\tilde{\mathcal{G}}$ associated to $\tilde{\sigma}$ is topologically principal.

Proof. Let $U$ be a nonempty open subset of $L$. We need to find $(x, t) \in U$ such that for all $k, l \geq 0$ if $\tilde{\sigma}^{k}(x, t)=\tilde{\sigma}^{l}(x, t)$ then $k=l$. Corollary 3.5 implies that if $\tilde{\sigma}^{k}(x, t)=\tilde{\sigma}^{l}(x, t)$ then $\sigma^{k}(x)=\sigma^{l}(x)$. Since $\pi(U)$ is open in $X$ there is $x \in \pi(U)$ such that for all $k, l \geq 0$ if $\sigma^{k}(x)=\sigma^{l}(x)$ then $k=l$. It follows that if we pick $t \in L_{x}$ such that $(x, t) \in U$ then $(x, t)$ satisfies the desired property. Hence $\tilde{\sigma}$ is essentially free and $\tilde{\mathcal{G}}$ is topologically principal.

Proposition 3.8. Let $\mathcal{G}$ be the Renault-Deaconu groupoid associated to the shift map $\sigma$ on $X$ and let $L$ be the fractafold bundle associated to a non-degenerate iterated function system $\left(F_{1}, \ldots, F_{N}\right)$ on a complete metric space $Y$. Let $\mathcal{G} * L$ be the left action groupoid defined via (3.2) and (3.3). Let $x \in X$ be an infinite word obtained by concatenating all the finite words in $W^{*}$. Then for all $t \in K \subset L_{x}$, the orbit of $(x, t) \in L \simeq(\mathcal{G} * L)^{0}$ is dense.

Proof. Recall (see, for example, [3, Theorem 4.2.1]) that every point $v$ in $K$ has at least one address $y \in X$, that is, $\{v\}=\bigcap_{n \geq 0} F_{y_{1}} \cdots F_{y_{n}}(K)$. Notice that the diameter of $F_{y_{1}} \cdots F_{y_{n}}(K)$ is less than $R_{y(n)} \cdot \operatorname{diam} K$ and recall that each $R_{i}$ is strictly smaller than 1 . We claim that the sequence $\left\{F_{x_{n}} \cdots F_{x_{1}}(t)\right\}_{n \in \mathbb{N}}$ is dense in $K$ for all $t \in K$. To prove the claim, let $v \in K$ and $\varepsilon>0$. There is $k \in \mathbb{N}$ and $\omega \in W^{k}$ such that $d\left(v, F_{\omega_{1}} \cdots F_{\omega_{k}}(u)\right)<\varepsilon$ for all $u \in K$. By hypothesis, $x$ contains the word $\omega_{k} \cdots \omega_{1}$. That is, there is $l \geq 1$ such that $x_{l}=\omega_{k}, \ldots$, $x_{l+k}=\omega_{1}$. Then

$$
d\left(v, F_{x_{l+k}} \cdots F_{x_{l}}\left(F_{x_{l-1}} \cdots F_{x_{1}}(t)\right)\right)=d\left(v, F_{\omega_{1}} \cdots F_{\omega_{k}}\left(F_{x_{l-1}} \cdots F_{x_{1}}(t)\right)\right)<\varepsilon
$$

for all $t \in K$. The claim follows.

We prove that the orbit of $(x, t) \in L_{0}$ is dense, where $t$ is an arbitrary point in $K$. Let $(y, v) \in L$ and let $U$ be a neighborhood of $(y, v)$ in $L$. Let $m \in \mathbb{N}$ and $\alpha \in W^{m}$ such that 
$(y, v) \in U_{m}:=U \cap\left(Z(\alpha) \times L_{m}(\alpha)\right)$. Then there is $V$ open in $K$ such that $(y, v) \in Z(\alpha) \times L_{m}^{\alpha}(V)$. We need to find $\gamma \in \mathcal{G}$ such that $\gamma \cdot(x, t) \in U$. Let $n \in \mathbb{N}$ be such that $F_{x(n)}(t) \in V$. Then $F_{\alpha(m)}^{-1} \circ F_{x(n)}(t) \in L_{m}^{\alpha}(V)$. Define $y \in X$ such that $y_{i}=\alpha_{i}, i=1, \ldots, m$ and $y_{m+i}=x_{n+i}$ for all $i \geq 1$. Then $(y, m-n, x) \in \mathcal{G}$ and $(y, m-n, x) \cdot(x, t) \in U$.

In general $L$ may fail to be locally compact (see Section 4 for specific examples of when this property fails). However, if $F_{i}(K)$ is open in $K$ for all $i=1, \ldots, N$, then $L$ is locally compact. In this case $K$ is a totally disconnected set. One can easily check that this condition is satisfied if the iterated function system is totally disconnected.

Suppose that $L$ is locally compact, then as observed at the end of Section $2, C^{*}(\mathcal{G} * L)$ is nuclear and a Cuntz-Pimsner algebra (where the correspondence is defined over $C_{0}(L)$ ). Proposition 3.8 allows us to conclude a bit more about $C^{*}(\mathcal{G} * L)$.

A $C^{*}$-algebra is defined to be primitive (see $[14, \S 3.13 .7]$ ) if it has a faithful irreducible representation.

Proposition 3.9. Assume that $F_{i}(K)$ is open in $K$ for all $i=1, \ldots, N$. With notation as in Proposition $3.8, C^{*}(\mathcal{G} * L)$ is primitive.

Proof. Let $z=(x, t) \in L \simeq(\mathcal{G} * L)^{0}$ be a point with dense orbit as guaranteed by Proposition 3.8. Note that point evaluation at $z$ defines a pure state on $C_{0}(L)$, the canonical masa (maximal abelian subalgebra) in $C^{*}(\mathcal{G} * L)$. Since it extends to a pure state on $C^{*}(\mathcal{G} * L)$, the GNS construction provides an irreducible representation $\pi_{z}$ of $C^{*}(\mathcal{G} * L)$ and a unit vector $\xi_{z}$ in the associated Hilbert space $H_{\pi_{z}}$ such that $\left\langle\pi_{z}(f) \xi_{z}, \xi_{z}\right\rangle=f(z)$ for all $f \in C_{0}(L)$. Since ker $\pi_{z} \cap C_{0}(L)$ is supported on an open set which does not contain any points in the orbit of $z$, $\operatorname{ker} \pi_{z} \cap C_{0}(L)=\{0\}$. By Corollary 2.3 and Proposition 3.7, $\mathcal{G} * L=\tilde{\mathcal{G}}$ is both amenable and topologically principal. Hence, every nonzero ideal in $C^{*}(\mathcal{G} * L)$ must have a nontrivial intersection with $C_{0}(L)$ by [10, Theorem 4.4] (see also [5, Proposition 5.5]). Hence, ker $\pi_{z}=\{0\}$ and thus $C^{*}(\mathcal{G} * L)$ has a faithful irreducible representation.

If $G$ is an étale groupoid, a $G$-invariant measure $\mu$ on $G^{0}$ is a measure such that for any open $G$-set $U, \mu(r(U))=\mu(s(U))$ (it suffices to prove this for basis of open $G$-sets). We show below that there is a $\mathcal{G} * L$-invariant measure $\mu_{\infty}$ on $L$ provided that the iterated function system satisfies one additional hypothesis. We will assume that the iterated function system $\left(F_{1}, \ldots, F_{N}\right)$ satisfies the open set condition [11, Definition 5.2.1]: there exists a non-empty open set $O$ such that $\cup_{i} F_{i}(O) \subset O$ and $F_{i}(O) \cap F_{j}(O)=\varnothing$ if $i \neq j$. A totally disconnected iterated function system satisfies the open set condition with $O=K$.

We will need to invoke a measure theoretic extension theorem (see [12, Theorem 6.2]) to extend a measure on a semialgebra of subsets of $L$ to the $\sigma$-algebra of Borel sets on $L$. Recall that a collection $\mathcal{C}$ of subsets of a set $\Omega$ is called a semialgebra if it is closed under finite intersections and if the complement of $B \in \mathcal{C}$ is expressible as a finite disjoint union of elements of $\mathcal{C}$. For each $n \in \mathbb{N}$, let $\mathcal{C}_{n}$ be the collection of Borel subsets of $L_{n}$. Then $\mathcal{C}=\left\{L_{n}^{c}: n \geq 1\right\} \cup\left(\bigcup_{n \in \mathbb{N}} \mathcal{C}_{n}\right)$ is a semialgebra.

By [11, Theorem 4.4.1], there is a unique Borel probability measure $\mu$ on $K$ such that

$$
\mu(A)=\frac{1}{N} \sum_{i=1}^{N} \mu\left(F_{i}^{-1}(A)\right)
$$

for all Borel subsets of $K$. Then, for each $n \in \mathbb{N}$ and $\omega \in W^{n}$ we can define a measure $\mu_{\omega}$ on $L_{n}(\omega)$ via $\mu_{\omega}(A)=N^{n} \mu\left(F_{\omega}(A)\right)$. We let $\nu$ be the product measure on $X$ generated by the weights $\{1 / N, \ldots, 1 / N\}$ on the set $\{1, \ldots, N\}$. Therefore, if $\omega \in W^{n}$ then $\nu(Z(\omega))=(1 / N)^{n}$. Then one can define a Borel measure $\mu_{n}$ on $L_{n}$ such that $\mu_{n}(Z(\omega) \times A)=\mu\left(F_{\omega}(A)\right)$, and, more 
generally, $\mu_{n}(U \times A)=\nu\left(\sigma^{n}(U)\right) \cdot \mu\left(F_{\omega}(A)\right)$ if $U \times A \subset Z(\omega) \times L_{n}(\omega)$. Notice that if $m<n$ and $B$ is a Borel subset of $L_{m}$ then $B$ is a Borel subset of $L_{n}$. However, more is true if the iterated function system satisfies the open set condition.

Lemma 3.10. Assume that the iterated functions system $\left(F_{1}, \ldots, F_{N}\right)$ on $T$ satisfies the open set condition. Then

1. The measure $\mu$ is the Hausdorff measure on $K$ and $\mu(A)=N \mu\left(F_{i}(A)\right)$ for all Borel sets $A \subset K$.

2. If $m<n$ and $B$ is a Borel subset of $L_{m}$ then $\mu_{n}(B)=\mu_{m}(B)$.

Proof. The first part is an immediate consequence of [11, Theorem 5.3.1]. The second part follows from the first part.

Proposition 3.11. Assume that the iterated function system $\left(F_{1}, \ldots, F_{N}\right)$ satisfies the open set condition. Then there is a unique $\tilde{\mathcal{G}}$-invariant Borel measure $\mu_{\infty}$ on $L$ such that $\mu_{\infty}\left(L_{n}^{c}\right)=\infty$ and

$$
\mu_{\infty}(U \times A)=\nu\left(\sigma^{n}(U)\right) \cdot \mu\left(F_{\omega}(A)\right)
$$

where $\omega \in W^{n}$ and $U \times A \subset Z(\omega) \times L_{n}(\omega)$ is Borel.

Proof. As noted above $\mathcal{C}=\left\{L_{n}^{c}: n \geq 1\right\} \cup\left(\bigcup_{n \in \mathbb{N}} \mathcal{C}_{n}\right)$ is a semialgebra. By [12, Theorem 6.2], $\mu_{\infty}$ extends uniquely to a measure on the $\sigma$-algebra generated by $\mathcal{C}$ if the following conditions hold

i. If $\varnothing \in \mathcal{C}, \mu_{\infty}(\varnothing)=0$.

ii. If $C=C_{1} \cup \cdots \cup C_{n}$ where $C, C_{1}, \ldots, C_{n} \in \mathcal{C}$ and $C_{i} \cap C_{j}=\varnothing$ for $i \neq j$, then $\mu_{\infty}(C)=$ $\mu_{\infty}\left(C_{1}\right)+\cdots+\mu_{\infty}\left(C_{n}\right)$.

iii. If $C \subset \bigcup_{n} C_{n}$ where $C, C_{1}, C_{2}, \ldots \in \mathcal{C}$, then $\mu_{\infty}(C) \leq \sum_{n} \mu_{\infty}\left(C_{n}\right)$.

iv. There are $C_{1}, C_{2}, \ldots \in \mathcal{C}$ such that $\mu_{\infty}\left(C_{n}\right)<\infty$ and $L=\bigcup_{n} C_{n}$.

Conditions (i) and (ii) are easy to check. The only non-trivial case for condition (iii) is when $C=L_{k}^{c}$ for some $k \in \mathbb{N}$, and for each $n \in \mathbb{N}$ there is $k_{n} \in \mathbb{N}$ such that $C_{n} \in \mathcal{C}_{k_{n}}$. Then $\mu_{\infty}(C)=\infty$ and $\lim _{k \rightarrow \infty} \mu_{\infty}\left(L_{k} \cap C\right)=\infty$. Let $k \in \mathbb{N}$ be fixed. Then the sets $L_{k} \cap C_{n}$ are Borel subsets of $L_{k}$ that cover $L_{k} \cap C$. Hence, using Lemma 3.10, we have that

$$
\mu_{k}\left(L_{k} \cap C\right) \leq \sum_{j} \mu_{k}\left(L_{k} \cap C_{j}\right) \leq \sum_{n} \mu_{\infty}\left(C_{n}\right)
$$

Since the left hand side of the inequality goes to $\infty$, it follows that $\sum_{n} \mu_{\infty}\left(C_{n}\right)=\infty=\mu_{\infty}(C)$.

To see that condition (iv) holds we first note that for each $\alpha \in W^{n}, C_{\alpha}=Z(\alpha) \times L_{n}(\alpha) \in \mathcal{C}$, $\mu\left(C_{\alpha}\right)=\mu(K)=1$ and $L=\bigcup_{\alpha \in W^{*}} C_{\alpha}$. It is straightforward to show that the $\sigma$-algebra generated by $\mathcal{C}$ is the Borels.

To prove that $\mu_{\infty}$ is $\tilde{\mathcal{G}}$-invariant we use the following fact that is probably known to specialists: if $\sigma$ is a local homeomorphism on a Hausdorff topological space $X$, then $\mathcal{G}(U, m, n, V)$ is a $\mathcal{G}$-section (where $\mathcal{G}=\mathcal{G}(X, \sigma)$ is the associated Renault-Deaconu groupoid), if and only if $\left.\sigma^{m}\right|_{U}$ is a homeomorphism onto $\sigma^{m}(U)$ and $\left.\sigma^{n}\right|_{V}$ is a homeomorphism onto $\sigma^{n}(V)$. Moreover, $r(\mathcal{G}(U, m, n, V))=U$ and $s(\mathcal{G}(U, m, n, V))=V$. 
Let $V$ be open in $L$ such that $\left.\tilde{\sigma}\right|_{V}$ is a homeomorphism onto $\tilde{\sigma}(V)$. It follows from the above remark that in order to show that $\mu_{\infty}$ is $\tilde{\mathcal{G}}$-invariant it is enough to prove that $\mu_{\infty}(V)=$ $\mu_{\infty}(\tilde{\sigma}(V))$. We have that

$$
\begin{aligned}
\mu_{\infty}(V) & =\mu\left(V \cap L_{0}\right)+\sum_{n \geq 0} \mu_{\infty}\left(V \cap\left(L_{n+1} \backslash L_{n}\right)\right) \\
& =\mu\left(V \cap L_{0}\right)+\sum_{n \geq 0} \sum_{\omega \in W^{n+1}} \mu\left(V \cap\left(Z(\omega) \times\left(L_{n+1}(\omega) \backslash L_{n}(\omega(n))\right)\right) .\right.
\end{aligned}
$$

Hence, it suffices to prove that if $\omega \in W^{n}$ and $U \times A \subset Z(\omega) \times L_{n}(\omega)$ is open such that $\left.\tilde{\sigma}\right|_{U \times A}$ is a homeomorphism onto $\tilde{\sigma}(U \times A)$, then $\mu(\tilde{\sigma}(U \times A))=\mu(U \times A)$. By the definition of $\tilde{\sigma}$ we have that $\tilde{\sigma}(U \times A)=\sigma(U) \times F_{\omega_{1}}(A)$. Hence, using Lemma 3.10,

$$
\mu_{\infty}(\tilde{\sigma}(U \times A))=\nu\left(\sigma^{n-1}(\sigma(U))\right) \cdot \mu\left(F_{\omega_{n} \cdots \omega_{2}}\left(F_{\omega_{1}}(A)\right)\right)=\mu_{\infty}(U \times A) .
$$

Thus $\mu_{\infty}$ is $\tilde{\mathcal{G}}$-invariant.

Corollary 3.12. Suppose that the iterated function system $\left(F_{1}, \ldots, F_{N}\right)$ satisfies the open set condition and that $F_{i}(K)$ is open in $K$ for all $i=1, \ldots, N$. With notation as above, the map

$$
\tau(f)=\int_{L} f d \mu_{\infty} \quad \text { for } \quad f \in C_{c}(\tilde{\mathcal{G}}) \cap C^{*}(\tilde{\mathcal{G}})^{+}
$$

extends to a densely defined lower semi-continuous trace on $C^{*}(\tilde{\mathcal{G}}) \simeq C^{*}(\mathcal{G} * L)$.

The proof of the corollary follows from the following lemma which is known to specialists. We were unable, however, to find a specific reference in the literature and we include a short proof for completeness.

Lemma 3.13. Suppose that $G$ is an étale locally compact groupoid and that $\mu$ is a (Radon) $G$ invariant measure on $G^{0}$. Then the map

$$
\tau(f)=\int_{G^{0}} f d \mu \quad \text { for all } f \in C_{c}(G) \cap C^{*}(G)^{+} .
$$

extends to a densely defined lower semi-continuous trace on $C^{*}(G)$.

Proof. Recall that for an étale locally compact groupoid $G$ the restriction map from $C_{c}(G)$ to $C_{c}\left(G^{0}\right)$ extends to a continuous expectation from $C^{*}(G)$ to $C^{*}\left(G^{0}\right)$ [16, Proposition II.1.15 and the remark following]. It is well known that a measure on a locally compact space $X$ induces a lower semi-continuous weight on $C_{0}(X)$ (see, for example, [4, Example II.6.7.2(v)]). Hence we obtain a densely defined lower semi-continuous weight $\tau$ on $C^{*}(G)$ by composition. Finally, since $\mu$ is invariant [16, Proposition II.5.4] implies that $\tau$ is a trace.

\section{Examples}

In this section we provide detailed descriptions of the fractafold bundle defined in Section 3 for some specific iterated function systems. We point out some cases when the bundle $L$ is not locally compact. We show in the second example that the action groupoid $\mathcal{G} * L$ is not, in general, minimal. 
Example 4.1. Let $F_{0}, F_{1}: \mathbb{R} \rightarrow \mathbb{R}$ be the maps $F_{0}(x)=\frac{1}{2} x$ and $F_{1}(x)=\frac{1}{2} x+\frac{1}{2}$. Then $\left\{F_{0}, F_{1}\right\}$ is an iterated function system whose invariant set is $[0,1]$. In this example, $W=\{0,1\}$, $X=W^{\infty}=\{0,1\}^{\infty}$ and the fractafolds defined in Section 3 have an easy description. Notice that, unlike in the previous section, we index our maps using 0 and 1 ; the index of an element in $X$ starts at 0 as well. This makes the formulas that we describe next more tractable. For $x \in X$ define $a_{n}(x)=F_{x(n)}^{-1}(0)$ and $b_{n}(x)=F_{x(n)}^{-1}(1)$ for $n \geq 1$. Then

$$
L_{n}(x)=\left[a_{n}(x), b_{n}(x)\right]
$$

and $L(x)$ is either the real line, a left half-closed infinite interval, or a right half-closed infinite interval. To prove this claim notice that $\left\{a_{n}(x)\right\}_{n}$ is a decreasing sequence and $\left\{b_{n}(x)\right\}_{n}$ is an increasing sequence. Indeed, if $x_{n}=0$ then $a_{n+1}(x)=a_{n}(x)$ and $b_{n}(x)<b_{n+1}(x)$, and if $x_{n}=1$ then $a_{n+1}(x)<a_{n}(x)$ and $b_{n}(x)=b_{n+1}(x)$. It follows that if there is $n \in \mathbb{N}$ such that $x_{i}=0$ for all $i \geq n$, then $L(x)=\left[a_{n}(x), \infty\right)$ and, if $x_{i}=1$ for all $i \geq n$, then $L(x)=\left(-\infty, b_{n}(x)\right]$. Otherwise $L(x)=\mathbb{R}$.

We claim that for any $x \in X$ we have that $a_{n}(x)=-\sum_{j=0}^{n-1} x_{j} 2^{j}$ and $b_{n}(x)=2^{n}-\sum_{j=0}^{n-1} x_{j} 2^{j}$ for all $n \geq 1$. In particular, $b_{n}(x)-a_{n}(x)=2^{n}$. One can prove this claim by induction as follows. Let $x \in X$. If $x_{0}=0$ then $a_{1}(x)=F_{0}^{-1}(0)=0$ and $b_{1}(x)=F_{0}^{-1}(1)=2$. If $x_{0}=1$ then $a_{1}(x)=F_{1}^{-1}(0)=-1$ and $b_{1}(x)=F_{1}^{-1}(1)=1$. Thus $a_{1}(x)=-x_{0} 2^{0}$ and $b_{1}(x)=2-x_{0} 2^{0}$ and the claim holds for $n=1$. Suppose that the induction hypothesis holds for all infinite words for $n \geq 1$. Then, if $x \in X$ and $z:=x_{1} x_{2} \cdots$, it follows that $a_{n}(z)=-\sum_{j=0}^{n-1} z_{j} 2^{j}=-\sum_{j=1}^{n} x_{j} 2^{j-1}$ and $b_{n}(z)=2^{n}-\sum_{j=0}^{n-1} z_{j} 2^{j}=2^{n}-\sum_{j=1}^{n} x_{j} 2^{j-1}$. If $x_{0}=0$ then

$$
a_{n+1}(x)=2 a_{n}(z)=-\sum_{j=1}^{n} x_{j} 2^{j}=-\sum_{j=0}^{n} x_{j} 2^{j}
$$

and

$$
b_{n+1}(x)=2 b_{n}(z)=2^{n+1}-\sum_{j=1}^{n} x_{j} 2^{j}=2^{n+1}-\sum_{j=0}^{n} x_{j} 2^{j} .
$$

If $x_{0}=1$ then

$$
a_{n+1}(x)=2 a_{n}(z)-1=-1-\sum_{j=1}^{n} x_{j} 2^{j}=-\sum_{j=0}^{n} x_{j} 2^{j}
$$

and

$$
b_{n+1}(x)=2 b_{n}(z)-1=2^{n+1}-1-\sum_{j=1}^{n} x_{j} 2^{j}=2^{n+1}-\sum_{j=1}^{n} x_{j} 2^{j} .
$$

Thus the induction holds and our claim is proved.

Therefore, for $n \in \mathbb{N}$ and $\alpha \in W^{n}$ we have that $L_{n}(\alpha)=\left[a_{n}(\alpha), b_{n}(\alpha)\right]$ and $L_{n}=\bigsqcup_{|\alpha|=n} Z(\alpha) \times$ $\left[a_{n}(\alpha), b_{n}(\alpha)\right]$. The local homeomorphism $\tilde{\sigma}$ on $L$ is

$$
\tilde{\sigma}(x, t)=\left\{\begin{array}{lll}
(\sigma(x), t / 2) & \text { if } & x_{0}=0 \\
(\sigma(x), t / 2+1 / 2) & \text { if } & x_{0}=1
\end{array}\right.
$$


Note that $L$ is not locally compact. For example, the point $(x, 0) \in L$ does not have a compact neighborhood, where $x$ is the sequence for which $x_{i}=0$ for all $i \in \mathbb{N}$. To see this, recall that if $C \subset L$ is compact then there is $n \geq 0$ such that $C \subset L_{n}$ (see [6, p. 2]). However, one can check that there is no open set in $L$ containing $(x, 0)$ that is a subset of any of the $L_{n}$ 's.

Example 4.2. Fix $N>1, r \in(0,1)$ and let $e_{1}, \ldots, e_{N} \in \mathbb{R}^{N}$ be the standard basis elements. For $j=1, \ldots, N$, define $F_{j}: \mathbb{R}^{N} \rightarrow \mathbb{R}^{N}$ by $F_{j}(x)=r x+(1-r) e_{j}$. Then $\left(F_{1}, \ldots, F_{N}\right)$ forms an iterated function system on $Y=\mathbb{R}^{N}$ (endowed with the usual metric). If $N=2$ and $r=1 / 2$, then the invariant set $K$ is homeomorphic to the unit interval and $\left(F_{1}, F_{2}\right)$ is conjugate to the iterated function system of the previous example. If $N=3$ and $r=1 / 2$ then $K$ is homeomorphic to the Sierpinski gasket.

We have $W=\{1, \ldots, N\}, X=W^{\infty}=\{1, \ldots, N\}^{\infty}$. Note that for $x \in X$ we have

$$
\lim _{k \rightarrow \infty} F_{x_{1}} \circ \cdots \circ F_{x_{k}}(0)=(1-r) \sum_{j=1}^{\infty} r^{j-1} e_{x_{j}} .
$$

Hence, the invariant set is given by $K=\left\{(1-r) \sum_{j=1}^{\infty} r^{j-1} e_{x_{j}}: x \in X\right\}$. It is straightforward to check that the iterated function system is totally disconnected if $r<1 / 2$.

Observe that $F_{j}^{-1}(x)=\frac{1}{r}\left(x-(1-r) e_{j}\right)$. For $\alpha \in W^{n}, L_{n}(\alpha)$ consists of all points of the form

$$
\frac{1-r}{r^{n+1}}\left(\sum_{j=1}^{\infty} r^{j} e_{x_{j}}-\sum_{i=1}^{n} r^{i} e_{\alpha_{i}}\right) .
$$

And, of course, for each $x \in X$, we have $L(x)=\bigcup_{n} L_{n}(x)$ (with the inductive limit topology).

We will show that the groupoid $\mathcal{G} * L$ is not minimal.

Let $y \in X$ be the sequence for which $y_{i}=1$ for all $i$. We claim that the orbit of $\left(y, e_{1}\right) \in L$ is not dense. In particular we show that $\left(y, e_{2}\right)$ is not in the closure of $\left\{\gamma \cdot\left(y, e_{1}\right): s(\gamma)=y\right\}$. If $s(\gamma)=y$, then $\gamma=(x, m-n, y)\left(\right.$ note $x_{i}=1$ for $\left.i \geq m\right)$. Since $e_{1}$ is a fixed point for $F_{1}$

$$
\gamma \cdot\left(y, e_{1}\right)=\left(x, F_{x(m)}^{-1} \circ F_{y(n)}\left(e_{1}\right)\right)=\left(x, F_{x(m)}^{-1}\left(e_{1}\right)\right)
$$

Define $f: \mathbb{R}^{N} \rightarrow \mathbb{R}$ by $f\left(t_{1}, \ldots, t_{N}\right)=t_{2}$. Note that $F_{j}^{-1}(t)=\frac{1}{r}\left(t-(1-r) e_{j}\right)$, so if $f(t) \leq 0$, then $f\left(F_{j}^{-1}(t)\right) \leq 0$. Observe that projection $\pi_{2}: L \rightarrow \mathbb{R}^{N}$ is continuous. An easy induction argument now shows that

$$
\left(f \circ \pi_{2}\right)\left(\gamma \cdot\left(y, e_{1}\right)\right)=f\left(F_{x(m)}^{-1}\left(e_{1}\right)\right) \leq 0 .
$$

Since $\left(f \circ \pi_{2}\right)\left(y, e_{2}\right)=1$ (and $f \circ \pi_{2}$ is continuous), $\left(y, e_{2}\right)$ is not in the closure of the orbit of $\left(y, e_{1}\right)$.

If $r<1 / 2$ the iterated function system is totally disconnected, and, hence, $F_{i}(K)$ is open in $K$. Thus, if $r<1 / 2, L$ is locally compact. However, if $r \geq 1 / 2$ then $L$ is not locally compact. An argument similar with the one at the end of Example 4.1 shows that, if $r \geq 1 / 2$, the point $\left(y, e_{1}\right) \in L$ does not have a compact neighborhood in $L$, where $y$ is the sequence for which $y_{i}=1$ for all $i \in \mathbb{N}$.

\section{Acknowledgements}

The work of the first author was partially supported by a grant from the Simons Foundation (\#209277 to Marius Ionescu). The authors would like to thank the referees for their helpful comments. 


\section{References}

[1] Anantharaman-Delaroche C., Purely infinite $C^{*}$-algebras arising from dynamical systems, Bull. Soc. Math. France 125 (1997), 199-225.

[2] Anantharaman-Delaroche C., Renault J., Amenable groupoids, in Groupoids in Analysis, Geometry, and Physics (Boulder, CO, 1999), Contemp. Math., Vol. 282, Amer. Math. Soc., Providence, RI, 2001, 35-46.

[3] Barnsley M.F., Fractals everywhere, 2nd ed., Academic Press Professional, Boston, MA, 1993.

[4] Blackadar B., Operator algebras. Theory of $C^{*}$-algebras and von Neumann algebras, operator algebras and non-commutative geometry, III, Encyclopaedia of Mathematical Sciences, Vol. 122, Springer-Verlag, Berlin, 2006.

[5] Brown J., Clark L.O., Farthing C., Sims A., Simplicity of algebras associated to étale groupoids, Semigroup Forum 88 (2014), 433-452, arXiv:1204.3127.

[6] Curtis D.W., Patching D.S., Hyperspaces of direct limits of locally compact metric spaces, Topology Appl. 29 (1988), 55-60.

[7] Deaconu V., Groupoids associated with endomorphisms, Trans. Amer. Math. Soc. 347 (1995), $1779-1786$.

[8] Deaconu V., Kumjian A., Muhly P., Cohomology of topological graphs and Cuntz-Pimsner algebras, J. Operator Theory 46 (2001), 251-264, math.OA/9901094.

[9] Edgar G., Measure, topology, and fractal geometry, 2nd ed., Undergraduate Texts in Mathematics, Springer, New York, 2008.

[10] Exel R., Non-Hausdorff étale groupoids, Proc. Amer. Math. Soc. 139 (2011), 897-907, arXiv:0812.4087.

[11] Hutchinson J.E., Fractals and self-similarity, Indiana Univ. Math. J. 30 (1981), 713-747.

[12] McDonald J.N., Weiss N.A., A course in real analysis, 2nd ed., Academic Press Inc., Amsterdam, 2013.

[13] Muhly P.S., Renault J.N., Williams D.P., Equivalence and isomorphism for groupoid $C^{*}$-algebras, J. Operator Theory 17 (1987), 3-22.

[14] Pedersen G.K., $C^{*}$-algebras and their automorphism groups, London Mathematical Society Monographs, Vol. 14, Academic Press, Inc., London - New York, 1979.

[15] Pimsner M.V., A class of $C^{*}$-algebras generalizing both Cuntz-Krieger algebras and crossed products by Z, in Free Probability Theory (Waterloo, ON, 1995), Fields Inst. Commun., Vol. 12, Amer. Math. Soc., Providence, RI, 1997, 189-212.

[16] Renault J., A groupoid approach to $C^{*}$-algebras, Lecture Notes in Mathematics, Vol. 793, Springer, Berlin, 1980.

[17] Renault J., Cuntz-like algebras, in Operator Theoretical Methods (Timişoara, 1998), Theta Found., Bucharest, 2000, 371-386, math.OA/9905185.

[18] Renault J., Cartan subalgebras in $C^{*}$-algebras, Irish Math. Soc. Bull. (2008), 29-63, arXiv:0803.2284.

[19] Strichartz R.S., Fractals in the large, Canad. J. Math. 50 (1998), 638-657.

[20] Strichartz R.S., Differential equations on fractals. A tutorial, Princeton University Press, Princeton, NJ, 2006. 